\title{
Systematic study of defect-related quenching of NV luminescence in diamond with time-correlated single-photon counting spectroscopy
}

\author{
D. Gatto Monticone, ${ }^{1,2,3,6}$ F. Quercioli,${ }^{4}$ R. Mercatelli, ${ }^{4}$ S. Soria,${ }^{5}$ S. Borini,,${ }^{7,}$ T. Poli, ${ }^{8}$ M. Vannoni,${ }^{4, \dagger}$ \\ E. Vittone, ${ }^{1,2,3,6}$ and P. Olivero ${ }^{1,2,3,6, \ddagger}$ \\ ${ }^{1}$ Physics Department, University of Torino, Torino, Italy \\ 2 “Nanostructured Interfaces and Surfaces" (NIS) Centre of Excellence, University of Torino, Torino, Italy \\ ${ }^{3}$ Istituto Nazionale di Fisica Nucleare (INFN), Sez. Torino, Torino, Italy \\ ${ }^{4}$ Consiglio Nazionale delle Ricerche (CNR), Istituto Nazionale di Ottica, Firenze, Italy \\ ${ }^{5}$ Consiglio Nazionale delle Ricerche (CNR), Istituto di Fisica Applicata "Nello Carrara," Firenze, Italy \\ ${ }^{6}$ Consorzio Nazionale Interuniversitario per le Scienze Fisiche della Materia (CNISM), sez. Torino, Italy \\ ${ }^{7}$ Thermodynamics Division, Istituto Nazionale di Ricerca Metrologica (INRiM), Torino, Italy \\ ${ }^{8}$ Chemistry Department, University of Torino, Italy
}

(Received 31 May 2013; revised manuscript received 30 August 2013; published 2 October 2013)

\begin{abstract}
We report on the systematic characterization of photoluminescence (PL) lifetimes in $\mathrm{NV}^{-}$and $\mathrm{NV}^{0}$ centers in 2-MeV H $\mathrm{H}^{+}$-implanted type Ib diamond samples by means of a time-correlated single-photon counting (TCSPC) microscopy technique. A dipole-dipole resonant energy transfer model was applied to interpret the experimental results, allowing a quantitative correlation of the concentration of both native (single substitutional nitrogen atoms) and ion-induced (isolated vacancies) PL-quenching defects with the measured PL lifetimes. The TCSPC measurements were carried out in both frontal (i.e., laser beam probing the main sample surface along the same normal direction of the previously implanted ions) and lateral (i.e., laser beam probing the lateral sample surface orthogonally with respect to the same ion implantation direction) geometries. In particular, the latter geometry allowed a direct probing of the centers lifetime along the strongly nonuniform damage profiles of $\mathrm{MeV}$ ions in the crystal. The extrapolation of empirical quasiexponential decay parameters allowed the systematic estimation of the mean quantum efficiency of the centers as a function of intrinsic and ion-induced defect concentration, which is of direct relevance for the current studies on the use of diamond color centers for photonic applications.
\end{abstract}

DOI: 10.1103/PhysRevB.88.155201

PACS number(s): 81.05.ug, 78.55.Qr, 61.72.jn

\section{INTRODUCTION}

The study of negatively charged single nitrogen-vacancy $\left(\mathrm{NV}^{-}\right)$luminescent centers in diamond has attracted a growing interest in recent decades, due to the opportunities they offer in the coherent manipulation of quantum states at room temperature, as well as in the efficient and high-rate emission of single photons on demand. Such unique properties make these centers appealing not only in fundamental quantum optics ${ }^{1-3}$ but also in advanced applications, such as quantum computing, ${ }^{4-6}$ single spin-based magnetic, electrical, and biological sensing ${ }^{7-9}$ quantum cryptography, ${ }^{10-12}$ and quantum nanomechanics. ${ }^{13-15}$

A key advantage of single luminescent centers in diamond such as the $\mathrm{NV}^{-}$complex is based on the fact that since they usually consist of deep defects in a wide band-gap material, they can be suitably considered as the solid-state analog of trapped atoms inside a spin-free environment characterized by a broad optical transparency. This is, of course, only true in an ideal crystal, and several works were devoted to assessing the variation of the spectral and spin coherence properties of the $\mathrm{NV}^{-}$center, depending on the concentration in the hosting crystal of structural defects, ${ }^{16,17}$ isotopic ${ }^{13} \mathrm{C}$ impurities, ${ }^{18-20}$ and foreign substitutional atoms such as nitrogen..$^{21,22}$

The important issue of achieving single $\mathrm{NV}^{-}$centers in bulk single-crystal diamond with minimum interaction with the surrounding crystal was addressed in a series of works with two approaches: optimizing the ion implantation and postimplantation processing ${ }^{23-30}$ and manipulating the $\mathrm{NV}^{-}$ centers with suitable noise-correcting procedures. ${ }^{31-37}$

Luminescence lifetime is an effective tool to directly study the nonradiative decay channels in color centers, thus giving significant information on their quantum efficiency, as much as on their interaction with the surrounding crystal environment. ${ }^{38,39}$ Luminescence lifetime studies have been applied in the characterization of defect interactions for various luminescent centers in diamond, such as the H3 (Refs. 40 and 41) and N3 (Ref. 42) centers. In both of the above-cited works, a significant decrease in lifetime was observed in samples characterized by high nitrogen concentrations.

In early works, the lifetime of $\mathrm{NV}^{-}$centers was evaluated as $(13 \pm 0.5) \mathrm{ns}$, although a relation between the centers lifetime and the crystal quality in synthetic samples was identified..$^{43,44}$

The $\mathrm{NV}^{-}$center is characterized by a triplet $(S=1)$ spin state, with different spin-projection states $\left(m_{S}=0\right.$ and $m_{S}= \pm 1$ ) being characterized by the same oscillator strength. However, intersystem crossing processes involving nonradiative transitions through intermediate metastable singlet states determine different lifetimes (13.7 and $7.3 \mathrm{~ns}$, respectively ${ }^{45}$ ) for the $m_{S}=0$ and $m_{S}= \pm 1$ spin-projection states of the defect, as found in lifetime measurements combined with MW manipulation of the excited states. ${ }^{45,46}$

Photoluminescence (PL) lifetime mapping measurements with a time-correlated single-photon counting (TCSPC) technique proved to be an effective tool to investigate the quenching mechanisms of $\mathrm{NV}^{-}$and $\mathrm{NV}^{0}$ centers. ${ }^{40,47}$ 
In the present paper, we report a systematic study on the variation of the lifetime of $\mathrm{NV}$ (i.e., $\mathrm{NV}^{-}$and $\mathrm{NV}^{0}$ ) centers as a function of ion-induced damage density in artificial high-pressure high-temperature (HPHT) diamonds with different nitrogen concentrations, performed with a TCSPC microspectroscopy technique.

\section{EXPERIMENTAL}

\section{A. Sample preparation and preliminary Fourier transform infrared characterization}

In the present study, three single-crystal type Ib diamond samples produced by Element Six with the HPHT technique were used. The sample size was $3 \times 3 \times 0.3 \mathrm{~mm}^{3}$. While samples 1 and 2 were optically polished on one of their two larger faces, sample 3 was optically polished on both frontal and lateral faces. Samples with a single growth sector were chosen, as observed by cross-polarization optical microscopy and infrared microscopy. The above-mentioned growth macrosectors developed during the HPHT synthesis from a single seed, and depending on their orientation, they are characterized by different impurity concentrations. ${ }^{48-50}$ Thus, it was assumed that the absence of observable sectors resulted in a uniform distribution of impurities within the sample.

The substitutional nitrogen concentration in the samples was characterized with micro-Fourier transform infrared ( $\mu$ FTIR) absorption transmission measurements, using a Bruker VERTEX 70 FT-IR spectrometer coupled to a Bruker Hyperion 3000 FT-IR optical microscope equipped with a mercury cadmium telluride (MCT) detector (InfraRed Associates, Inc.) in the spectral range of 4000 to $600 \mathrm{~cm}^{-1}$, with an average spectral resolution of $4 \mathrm{~cm}^{-1}$.

In Fig. 1, typical $\mu$-FTIR spectra obtained from samples 1-3 are reported. Sample 1 is characterized by the lowest nitrogen concentration; therefore, the absorption feature at wave number $1 / \lambda=1130 \mathrm{~cm}^{-1}$ (indicated by the black

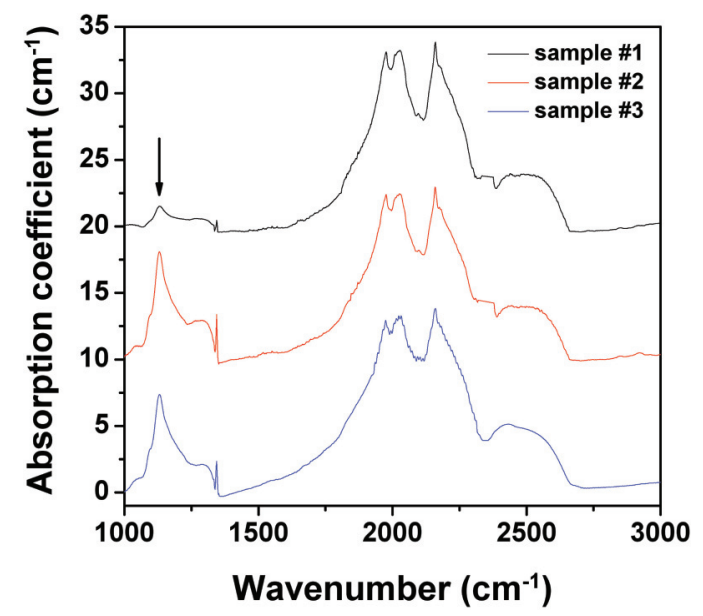

FIG. 1. (Color online) The $\mu$-FTIR spectra from samples 1 (black line), 2 (red line), and 3 (blue line) before ion implantation. The spectra from samples 1 and 2 are displaced along the vertical axis by $10 \mathrm{~cm}^{-1}$ and $20 \mathrm{~cm}^{-1}$ for sake of clarity. The spectrum from sample 3 is correctly referred to the vertical axis in terms of absolute values. The black arrow indicates the $1130-\mathrm{cm}^{-1}$ feature associated with the absorption from single substitutional nitrogen (Ref. 51). arrow) is less pronounced. On the other hand, samples 2 and 3 are characterized by similar nitrogen concentrations. The assumption of the homogeneity of samples was qualitatively confirmed by probing the samples at 10 different locations ( $20 \times 20 \mu \mathrm{m}^{2}$; each close to one of the implanted areas), and $\sim 10 \%$ variations on measured spectra were observed. The absorption coefficient at $1 / \lambda=1130 \mathrm{~cm}^{-1}$ was measured to estimate the concentration of single substitutional nitrogen $\left[N_{S}\right]$, adopting the calibration reported in Ref. 51. The resulting estimates of $\left[N_{S}\right]$ are $(0.60 \pm 0.05) \times 10^{2} \mathrm{ppm}$, $(2.0 \pm 0.2) \times 10^{2} \mathrm{ppm}$, and $(1.9 \pm 0.3) \times 10^{2} \mathrm{ppm}$ for samples $1-3$, respectively.

\section{B. Ion implantation and postprocessing}

Samples were implanted at room temperature across the polished surface with $2-\mathrm{MeV} \mathrm{H}^{+}$ions at the AN2000 microbeam facility of the Legnaro National Laboratories with a raster-scanning ion microbeam focused to a size of $\sim 5 \mu \mathrm{m}$, in order to deliver a uniform fluence across the irradiated areas. While samples 1 and 2 were implanted across the frontal surface in square areas $\left(100 \times 100 \mu \mathrm{m}^{2}\right.$ and $200 \times 200 \mu \mathrm{m}^{2}$ ) at different fluences, ranging from $5 \times 10^{14} \mathrm{~cm}^{-2}$ to $1 \times 10^{17} \mathrm{~cm}^{-2}$ (subsequently referred to as "frontal implantations"), sample 3 was implanted in two areas across its edge at fluences $1 \times 10^{15} \mathrm{~cm}^{-2}$ and $2 \times 10^{17} \mathrm{~cm}^{-2}$, with the purpose of performing cross-sectional optical characterization in a lateral geometry, as schematically shown in Fig. 2 (subsequently referred to as "lateral implantations"). In all implantations, beam current was $\sim 0.5 \mathrm{nA}$. Figure 3 shows the strongly nonuniform depth profile of the linear damage density, as evaluated with the Stopping and Range of Ions in Matter (SRIM)-2008.04 Monte Carlo code ${ }^{52}$ in "detailed calculation with full damage cascade" mode by taking an atom displacement energy value of $50 \mathrm{eV} .{ }^{53}$

After ion implantation, the samples were thermally annealed in vacuum $\left(p<10^{-4} \mathrm{~Pa}\right)$ for $2 \mathrm{~h}$ at a temperature of $800^{\circ} \mathrm{C}$, which is considered suitable for the conversion to

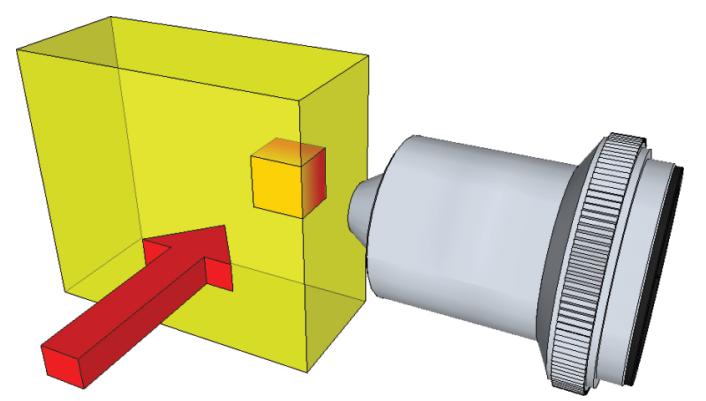

FIG. 2. (Color online) Schematic representation of the ion implantation performed on sample 3 with the purpose of allowing cross-sectional optical characterization in a lateral geometry. The yellow parallelogram represents the diamond sample, with one of its two main faces frontally exposed to ion implantation; the red arrow indicates the direction of the incoming ion beam, which is irradiating a square area (highlighted in orange within the sample) across the sample edge. The optical objective on the right side represents the optical setup, which is scanning the sample orthogonally with respect to the irradiation direction. The drawing is not to scale. 


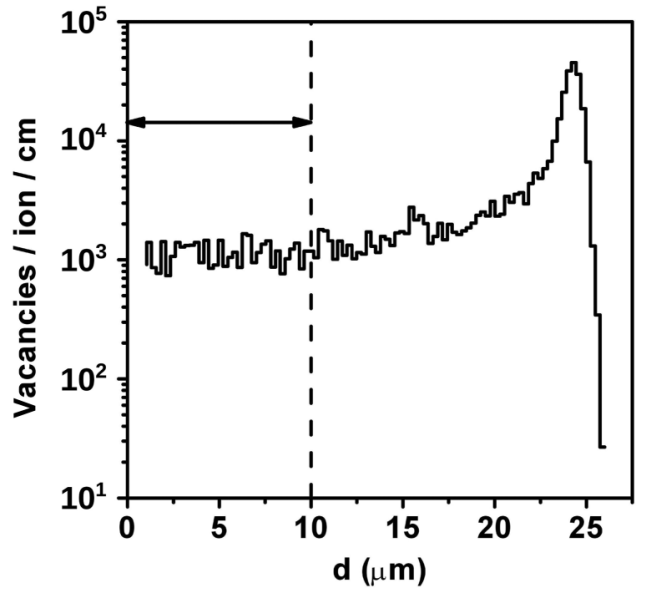

FIG. 3. Depth profile of the damage linear density, as evaluated from SRIM-2008.04 Monte Carlo code (Ref. 52). The damage density is parameterized in number of vacancies per incoming ion and unit length in the depth direction. The vertical dashed line highlights the probing depth of the TCSPC microscopy technique.

$\mathrm{NV}$ centers of a large fraction of the ion-induced vacancies and of the native nitrogen. ${ }^{54}$

\section{PL characterization}

Room temperature PL spectroscopy was performed for the purpose of preliminarily assessing the spectral features of the induced NV luminescence in the samples. A Jobin Yvon Raman microspectrometer was used for this scope, with 532-nm laser excitation and a charge-coupled device Andor DU420A-OE detector. Subsequent PL spectra from each sample are mutually comparable in terms of absolute PL intensities within a reasonable level of confidence, since particular care was taken in carrying the measurements within a reasonably short time frame in the same experimental conditions, particularly for the surface focusing procedure. Figures 4(a) and 4(b) show the PL spectra collected from the different implanted areas of samples 1 and 2, respectively. In

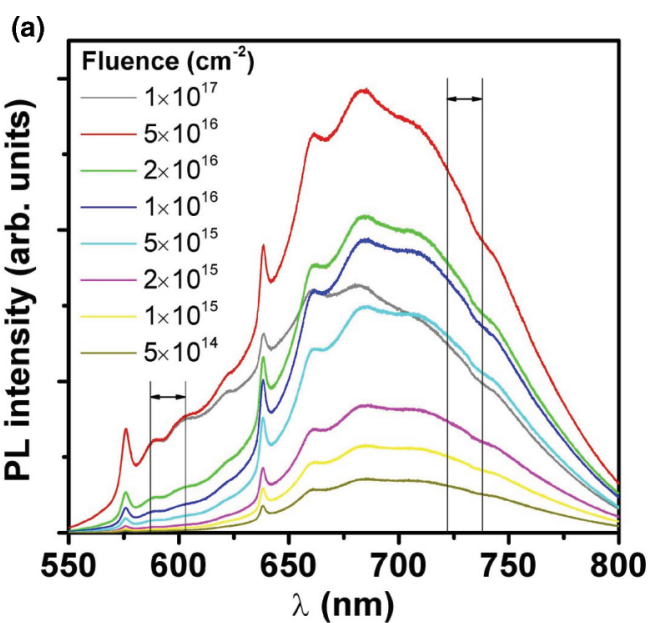

the spectra, the $\mathrm{NV}^{-}$[zero phonon line (ZPL): $\lambda=638 \mathrm{~nm}$ ] and $\mathrm{NV}^{0}$ (ZPL: $\lambda=575 \mathrm{~nm}$ ) emissions are visible together with their respective phonon sidebands, while no emission related to single vacancies (ZPLs: $\lambda=740.9 \mathrm{~nm}$ and $744.4 \mathrm{~nm}$; also referred as "GR1") is visible. The latter evidence provides a quantitative indication that after $800^{\circ} \mathrm{C}$ annealing, a large fraction of induced vacancies recombined with native nitrogen to form NV complexes.

The PL emission from sample 1 is characterized by a lower $\mathrm{NV}^{-}: \mathrm{NV}^{0}$ emission ratio with respect to sample 2 , in which $\mathrm{NV}^{0}$ emission is very weak. This can be explained if it is considered that in low-nitrogen samples, the $\mathrm{NV}$ centers are less likely to be in proximity with nearby donors and therefore are more probably found in their neutral charge state; on the other hand, $\mathrm{NV}^{-}$emission is predominant in samples characterized by high nitrogen concentration. ${ }^{55}$ Also, it is worth noting that consistent with previous reports, ${ }^{16}$ the evolution of the overall PL intensity as a function of implantation fluence follows a nonmonotonic trend. This can be explained by considering the effect of the increasing defect concentration induced by higher fluence implantations. At low fluences, this causes an initial increase of PL yield as more and more luminescent centers are generated, while at high fluences, an increasing defect density starts being detrimental to the PL yield, due to the combined effects of damage-induced optical absorption and nonradiative coupling with the existing luminescent centers (i.e., quenching). The latter process is systematically investigated in the present work by means of the TCSPC technique.

The intensities of the $\mathrm{NV}^{-}$and $\mathrm{NV}^{0} \mathrm{ZPL}$ emissions were evaluated by spectral integration after suitable background subtraction, and in Fig. 5, the evolution as a function of implantation fluence of the $\left(\mathrm{NV}^{-}: \mathrm{NV}^{0}\right)$ ratio is shown. At increasing fluences, the $\left(\mathrm{NV}^{-}: \mathrm{NV}^{0}\right)$ ratio exhibits a monotonically decreasing trend in both samples. This observation is compatible with previous reports ${ }^{16}$ and has several possible explanations, which are not mutually exclusive: (i) a decrease in the concentration of negatively charged NV centers due to the lowering of the Fermi level caused by the conversion of

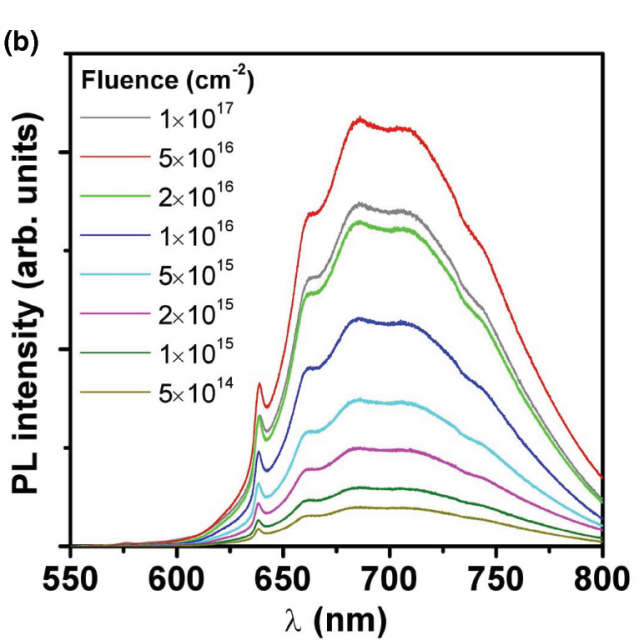

FIG. 4. (Color online) Room-temperature PL spectra from samples 1 (a) and 2 (b) after ion implantation and thermal annealing. Different spectra correspond to different implantation fluences, as indicated in the legends. Both $\mathrm{NV}^{-}(\mathrm{ZPL}: \lambda=638 \mathrm{~nm})$ and NV$(\mathrm{ZPL}: \lambda=575 \mathrm{~nm})$ emissions are visible, while no GR1 (ZPLs: $\lambda=740.9 \mathrm{~nm}$ and $744.4 \mathrm{~nm})$ emission is observed. The $(730 \pm 8 \mathrm{~nm})$ and $(595 \pm 8 \mathrm{~nm}) \mathrm{spectral}$ intervals adopted in TCSPC measurements are highlighted in (a). 


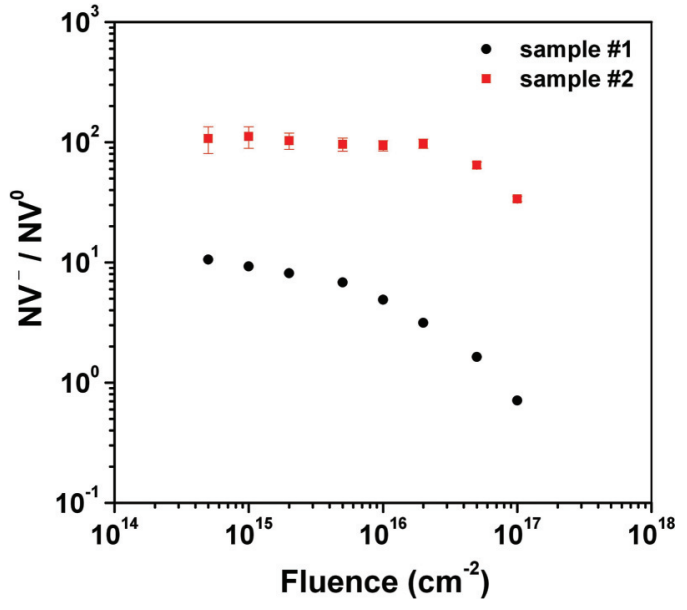

FIG. 5. (Color online) Evolution of the $\left(\mathrm{NV}^{-} / \mathrm{NV}^{0}\right) \mathrm{ZPL}$ emission ratio as a function of implantation fluence for samples 1 (black circles) and 2 (red squares). In the data relevant to sample 1, the uncertainty bars are smaller than the symbol size and have, therefore, not been reported.

native nitrogen atoms into NV complexes, (ii) a decrease in the concentration of negatively charged NV centers due to the electron-trapping effect of induced defects, and (iii) a more effective nonradiative coupling of induced defects with the $\mathrm{NV}^{-}$centers with respect to the $\mathrm{NV}^{0}$ centers.

\section{TCSPC measurements}

In the TCSPC measurements, the luminescence excitation was provided by a tunable Ti:Sapphire mode-locked laser source (700-890 nm; Coherent Mira 900-F) pumped by a frequency-doubled $\mathrm{Nd}: \mathrm{YVO}_{4}$ laser emitting at $532 \mathrm{~nm}(5 \mathrm{~W}$ CW; Coherent Verdi V5). The laser emission was modulated with a cavity dumper (A.P.E Berlin PulseSwitch) in order to obtain a pulse repetition rate of $10 \mathrm{MHz}$. The pulse duration is of the order of $10^{2}$ fs. The excitation laser light was sent into a microstructured fiber (NL-1.7-670 crystal fiber) for supercontinuum generation, and the excitation band was selected by a (485 $\pm 12 \mathrm{~nm}$ ) bandpass filter (HQ485/25 M, Chroma Technology Corporation). The excitation light was then coupled into a Nikon PCM2000 confocal laser scanning microscope unit equipped with a Nikon TE2000-U inverted optical microscope. In all measurements, the microscope was equipped with a Nikon $40 \times$ Plan Fluor objective [numerical aperture (NA) $=0.75$, air], with the exception of the measurements performed on sample 3, in which a Nikon $60 \times$ Plan Fluor $(\mathrm{NA}=1.4$, oil) was used with no coverslip between the lens and the sample. The microscopy system had a typical lateral spatial resolution of $\sim 1 \mu \mathrm{m}$, while the probe depth of the system was estimated to be $\sim 10 \mu \mathrm{m}$. In Fig. 3, the probing depth is highlighted, showing that in frontally implanted samples a constant damage density is probed.

The fluorescent light was collected into a single-grating spectrometer (Oriel Fics 77442) equipped with a 405 grooves $/ \mathrm{mm}$ grating and then detected with a Becker \& Hickl PML-16 multichannel head containing a 16-channel photomultiplier tube (Hamamatsu R5900-L16) and the relevant electronics. The signal is then processed with the TCSPC technique that allows the measurement of the fluorescence decay curve for each pixel of the acquired image. The core element of the TCSPC system is the SPC-830 module installed on a single printed circuit board. The TCSPC technique is based on the measurement of the arrival times of individual photons with respect to the excitation pulse and on the subsequent statistical reconstruction of the decay curve from multiple single-photon measurements, on a pixel-by-pixel basis. ${ }^{56}$ The time window of the acquired chronograms is $100 \mathrm{~ns}$, with a 256 time-bin resolution.

From a spectral point of view, it is possible to collect the fluorescence in the $\lambda=498-738 \mathrm{~nm}$ range, which is subdivided into 16 spectral channels. In the present work, the $(730 \pm 8 \mathrm{~nm})$ and the $(595 \pm 8 \mathrm{~nm})$ spectral intervals were used to acquire, respectively, the PL signal from the phonon sidebands of the $\mathrm{NV}^{-}$and $\mathrm{NV}^{0}$ emissions, as shown in Fig. 4(a). The collection of the PL signals at the phonon sidebands allowed a significant increase in signal statistics due to strong phonon coupling in NV centers, where only a small fraction of the PL emission is concentrated in the ZPL. More importantly, the $(730 \pm 8 \mathrm{~nm})$ spectral interval for $\mathrm{NV}^{-}$ detection was chosen to avoid spectral overlap with the tail of the $\mathrm{NV}^{0}$ phonon sidebands, while retaining significant signal intensity from the phonon sideband of the $\mathrm{NV}^{-}$emission. In a series of preliminary tests, wherever possible (i.e., where no significant spectral overlap occurred), it was verified that the lifetime behavior of ZPL and phonon-sideband emissions are mutually consistent, for both kinds of centers.

In general, PL decay chronograms relevant to regions implanted at different fluences in samples 1 and 2 were acquired by grouping the signals acquired from different pixels within each implanted region in order to improve signal statistics. The Gaussian shape of the scanning ion microbeam used to carry the implantation determines a lack of sharpness at the edges of the irradiated regions; therefore, care was taken to avoid pixels being too close to the edges of the implanted regions [see Fig. 6(a)].

The temporal response function of the instrument (defined as a convolution of the finite duration of the excitation pulse and of the time resolution of the acquisition system) was determined by measuring the duration of the excitation pulse, which was leaking through the monochromator at the $(506 \pm 8 \mathrm{~nm})$ spectral channel, resulting in a Gaussian pulse with a FWHM of $780 \mathrm{ps}$, corresponding to two of the 256 time bins into which the 100-ns temporal window was subdivided.

\section{THEORY}

It is well known that the luminescent emission of an unperturbed excited photoactive system (such as an ideal single NV center in a perfect diamond crystal) has an exponential time dependence characterized by a specific decay rate. The presence of a nearby system (such as a structural defect or an impurity in the diamond crystal, generally referred as a "quencher" of the optical transition under investigation) to which the luminescent center is resonantly coupled determines the appearance of a new nonradiative decay channel for the center. Therefore, the photon emission rate $I(t)$ is defined in this case as

$$
I(t)=-\frac{d N(t)}{d t}=a \cdot N_{0} \cdot \exp [-(a+K) \cdot t],
$$



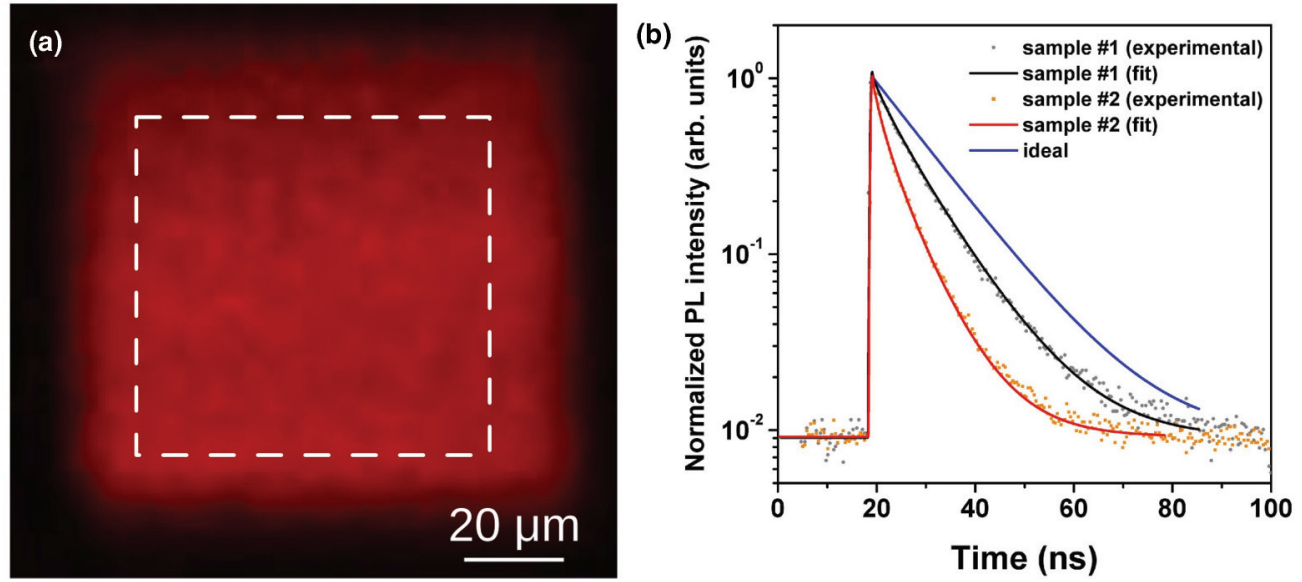

FIG. 6. (Color online) (a) Typical TCSPC map acquired in the $(730 \pm 8 \mathrm{~nm})$ spectral window (corresponding to the phonon sideband of the $\mathrm{NV}^{-}$emission) from a $100 \times 100 \mu \mathrm{m}^{2}$ region of sample 2 implanted with $2-\mathrm{MeV} \mathrm{H}^{+}$ions at a fluence of $1 \times 10^{16} \mathrm{~cm}^{-2}$; the region from which chronograms are extracted and analyzed is highlighted by the white dashed square. (b) Typical chronograms of normalized PL intensity acquired in the $\left(730 \pm 8 \mathrm{~nm}\right.$ ) spectral window from a region of sample 1 implanted at a fluence of $1 \times 10^{16} \mathrm{~cm}^{-2}$ (gray circles: experimental data, black line: fit); sample 2 implanted at a fluence of $1 \times 10^{16} \mathrm{~cm}^{-2}$ (orange squares: experimental data, red line: fit); an ideal curve corresponding to no luminescence quenching (i.e., $k=0$ ) is also reported for comparison (blue line).

where $N$ is the number of excited luminescent centers, $a$ is the intrinsic radiative decay rate of the luminescent center, and $K$ is the nonradiative decay rate associated with the resonant coupling with the nearby defect/impurity. The latter term depends from the strength of the resonant coupling, which, in turn, depends from the distance between the two systems. A dipoledipole coupling determines a dependence from the sixth power of the distance, while a dipole-quadrupole coupling determines a dependence from the eighth power of the distance and so on.

In a more realistic case, the luminescent center is coupled to a large number of nearby defects/impurities located at different distances. According to the theory developed in Refs. 57, 38, and 39, an integration of the different resonant coupling strengths for randomly located quenchers around the luminescent center results in the following deformed exponential decay rate:

$$
I(t)=a \cdot N_{0} \cdot \exp (-a \cdot t) \cdot \exp \left[-k \cdot(a \cdot t)^{c}\right],
$$

where $k$ is now a dimensionless parameter dependent from the quenchers concentration and the intensity of the resonant coupling, while the value of $c$ depends on the nature of the resonant coupling $(c=1 / 2$ for dipole-dipole interaction and $c=3 / 8$ for dipole-quadrupole interaction).

The mean quantum efficiency $\eta$ of luminescent centers surrounded by a given quencher distribution can be derived as the ratio between its total number of radiative decays [obtained by integrating Eq. (2)] and the total number of radiative decays of a corresponding isolated center (obtained by integrating a simple exponential function). Therefore, in the case of dipole-dipole coupling, the quantum efficiency $\eta$ can be derived as a function of the "coupling strength" parameter $k$ as follows: ${ }^{38}$

$$
\eta(k)=\frac{1}{2} \cdot\left\{2+\sqrt{\pi} \cdot k \cdot \exp \left(\frac{k^{2}}{4}\right) \cdot\left[\operatorname{erf}\left(\frac{k}{2}\right)-1\right]\right\},
$$

where erf is the Gaussian error function.
With more specific reference to our system of interest (i.e., NV centers in a defect and impurities containing diamond lattice), some additional preliminary considerations can be formulated based on the information available in literature.

Substitutional nitrogen defects behave as quenchers of the NV emission, as much as of other luminescent centers. ${ }^{41,42}$ As mentioned above, evidence of dipole-dipole resonant energy transfer from $\mathrm{NV}^{-}$centers to single substitutional nitrogen in an artificial diamond was reported in early works ${ }^{43,44}$ and more recently investigated with the TCSPC technique. ${ }^{40}$ In Ref. 40, also A aggregates (i.e., $\mathrm{N}_{2}$ complexes) were identified as effective quenchers of $\mathrm{NV}^{-}$emission, while $\mathrm{B}$ aggregates (i.e., $\mathrm{N}_{4}$ complexes) do not appear as effective in this respect. In a subsequent work, also the dipole-dipole resonant energy transfer from $\mathrm{NV}^{0}$ centers to neutral substitutional nitrogen was investigated in detail. ${ }^{47}$

Several previous works report extensively about the effect on the intensity of $\mathrm{NV}$ centers of structural defects induced from neutron, ${ }^{58}$ electron, ${ }^{59}$ and ion $^{16}$ irradiation, and it is natural to assume that also the center lifetime is affected by radiation-induced damage. While a $2-\mathrm{MeV}$ electron irradiation at a fluence of $2 \times 10^{18} \mathrm{~cm}^{-2}$ does not seem to have significant effects on the center lifetime, ${ }^{40}$ in the present work we report a systematic investigation on the effect of ion implantation on NV center lifetime in samples characterized by different substitutional nitrogen concentrations.

\section{EXPERIMENTAL RESULTS}

\section{A. Data analysis}

Figure 6(a) shows a typical TCSPC map acquired in the $(730 \pm 8 \mathrm{~nm}$ ) spectral window (corresponding to the phonon sideband of the $\mathrm{NV}^{-}$emission) from a $100 \times 100 \mu \mathrm{m}^{2}$ region of sample 2 implanted with $2-\mathrm{MeV} \mathrm{H}^{+}$ions at a fluence of $1 \times 10^{16} \mathrm{~cm}^{-2}$. The intensity map clearly highlights the implanted region where a higher concentration of $\mathrm{NV}$ centers is 
formed. It is worth stressing that apart from PL intensity, each pixel encodes a PL decay chronogram. The typical region from which chronograms are extracted and analyzed is highlighted by the white dashed square.

Figure 6(b) shows chronograms of normalized PL intensity acquired in the $(730 \pm 8 \mathrm{~nm})$ spectral window from regions of samples 1 and 2 implanted at fluences of $1 \times 10^{16} \mathrm{~cm}^{-2}$. The experimental data are plotted together with the relevant fitting curves with Eq. (2) by adopting $c=1 / 2$. The different decay trends are clearly distinguishable, with $k$ values corresponding to $(0.65 \pm 0.02)$ and $(1.80 \pm 0.03)$ for samples 1 and 2 , respectively. The corresponding quantum efficiency values are 58 and $27 \%$, respectively. For sake of comparison, an ideal curve corresponding to no luminescence quenching (i.e., $k=0, \eta=100 \%$ ) is also reported with the same background signal (blue line). The quality of the data fitting is very satisfactory, with reduced $\chi^{2}$ values of 1.3 and 1.5 , respectively, thus confirming the validity of the previously described model. A fit with Eq. (2) using the dipole-quadrupole coupling (i.e., $c=3 / 8$ ) was less satisfactory for both $\mathrm{NV}^{0}$ and $\mathrm{NV}^{-}$emissions, as reported in previous works. ${ }^{47}$ This result, combined with the fact that both NV centers and single substitutional nitrogen have electric dipoles, supports the hypothesis of dipole-dipole coupling.

Concerning the data analysis procedure, it is worth remarking that in all fitting procedures, the uncertainties on the photon counts have been estimated according to Poissonian statistics and that the temporal response function of the instrument has been suitably taken into account. As for the radiative lifetime constants, we assumed that in our optical excitation conditions (laser pulse wavelength, intensity, and duration) an efficient initialization of the $\mathrm{NV}^{-}$centers in the $m_{S}=0$ state $^{60}$ was obtained, despite the fact that optically induced spin polarization in $\mathrm{NV}^{-}$centers is indeed limited to $\sim 80 \% .^{45,61,62}$ This approximation was motivated by the fact that the resonant coupling strengths for the different types of transitions are unknown, as much as the exact degree of spin polarization of the defects in our system, and introducing all of the above-mentioned quantities as free parameters would have determined an excessive degree of mutual correlations in the fitting procedure. However, it was observed that the degree of uncertainty introduced by assuming a full $m_{S}=0$ spin polarization of the $\mathrm{NV}^{-}$system is of the same order of magnitude (i.e., $\sim 5-10 \%$ ) as the reported uncertainties on the resulting $k$ values. Therefore, in all fitting procedures a (12 $\pm 1 \mathrm{ns)} \mathrm{lifetime} \mathrm{corresponding}$ to the $m_{S}=0$ spin state was set for the $\mathrm{NV}^{-}$decays. ${ }^{46}$ The radiative lifetime of $\mathrm{NV}^{0}$ centers was preliminarily estimated as $(17 \pm 1 \mathrm{~ns})$ in an optical-grade chemical vapor deposition (CVD) diamond from Element Six that was implanted with $10-\mathrm{MeV} \mathrm{N}$ ions at a fluence of $2 \times 10^{13} \mathrm{~cm}^{-2}$ and subsequently annealed with the same procedure described above. As reported in Fig. 7, the decay was purely exponential within experimental uncertainty; therefore, we conclude that in the above-mentioned sample the luminescent centers could be considered (within the sensitivity limit of our technique) as free from quenching effects. The obtained value represents one of the few estimations of radiative lifetime in $\mathrm{NV}^{0}$ centers available in literature, in satisfactory agreement with previous results. ${ }^{47}$

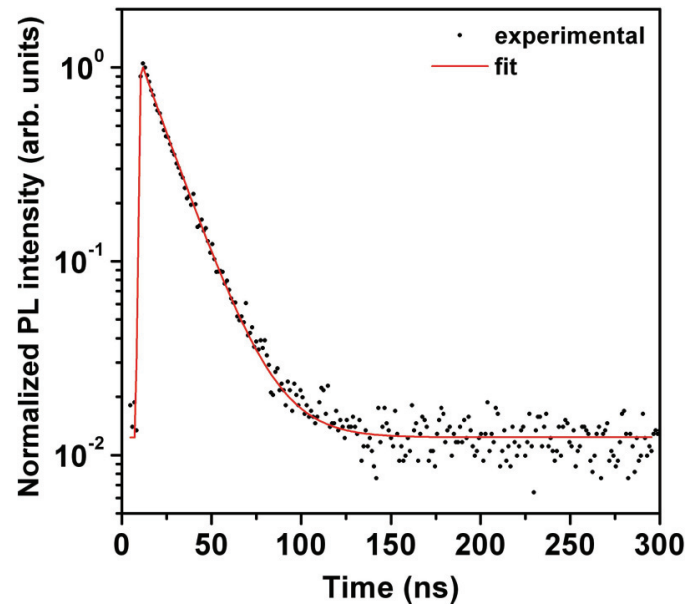

FIG. 7. (Color online) Chronogram of normalized $\mathrm{NV}^{0} \mathrm{PL}$ intensity acquired in the $(595 \pm 8 \mathrm{~nm})$ spectral window from an optical grade CVD diamond sample after $10-\mathrm{MeV} \mathrm{N}$ implantation at $2 \times 10^{13} \mathrm{~cm}^{-2}$ fluence and subsequent $800^{\circ} \mathrm{C}$ annealing. Experimental data (black circles) are reported with an exponential fitting curve (red line) from which a lifetime of $17 \pm 1 \mathrm{~ns}$ is resulting.

\section{B. Frontal implantations}

As mentioned above, for samples 1 and 2 optical probing was performed in the same geometry as the ion implantations, i.e., perpendicularly to the main surface of the sample. This approach resulted in an optical measure arising from the integration along the implantation depth over a thickness corresponding to the probing depth of the technique $(\sim 10 \mu \mathrm{m})$. The implantation of areas at different fluences in the two samples allowed a systematic study of the variation of the NV lifetimes as a function of induced damage densities, which were parameterized in a simple linear approximation (i.e., by ignoring nonlinear damage effects such as defect-defect interactions and self-annealing processes) by multiplying the vacancy linear density profile resulting from SRIM simulations (see Fig. 3) by the implantation fluence, thus obtaining a "parametric" vacancy density. Moreover, it is worth stressing that the reported vacancy density estimations are relevant to the samples prior to thermal annealing; therefore, they cannot be accounted as a realistic estimation of the physical vacancy density in the samples under analysis. For each implantation fluence, a mean vacancy density was estimated by averaging the vacancy density profile across the probing depth of the technique.

Figures 8(a) and 8(b) show the evolution of $k$, as evaluated from the fitting of relevant decay chronograms [see Fig. 6(b)], for the $\mathrm{NV}^{0}$ and $\mathrm{NV}^{-}$emissions (respectively) from sample 1 as a function of $2-\mathrm{MeV} \mathrm{H}^{+}$implantation fluence. The above-mentioned mean vacancy density is also reported in the upper horizontal axis, while corresponding quantum efficiency values [as evaluated from the value of $k$ with Eq. (3)] are reported on the right-hand vertical axis in a nonlinear scale. In both cases, the variation of the $k$ parameter follows a nonmonotonic trend as a function of damage density.

As for the $\mathrm{NV}^{0}$ emission data shown in Fig. 8(a), the reported $k$ vs fluence trend can be interpreted as follows. The initial decrease in the $k$ value at low fluences is because a significant fraction of the induced vacancies is recombining 
(a)

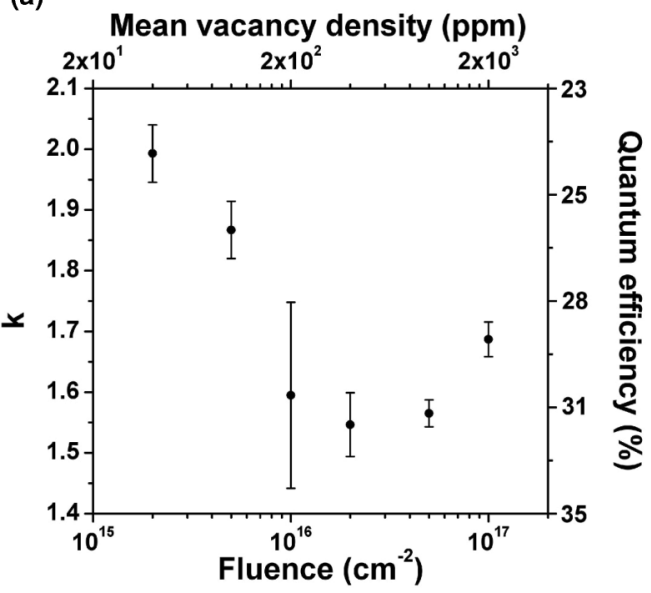

(b)

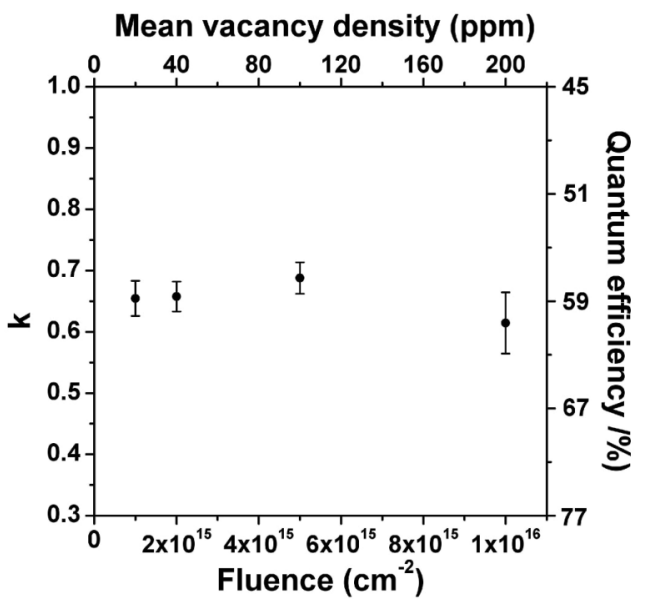

FIG. 8. Evolution of $k$ for the $\mathrm{NV}^{0}$ (a) and $\mathrm{NV}^{-}$(b) emissions from sample 1 as a function of 2-MeV $\mathrm{H}^{+}$implantation fluence. The two graphs are reported with the same extension (i.e., $\Delta k=0.7)$ in the left vertical axis for comparison. The mean vacancy density across the technique probing depth is reported in the upper horizontal axis, while corresponding quantum efficiency values [as derived from Eq. (3)] are reported on the right-hand vertical axis in a nonlinear scale.

with native nitrogen atoms, thus increasing the population of NV centers in the sample. Therefore, it is reasonable to assume that, at increasing fluences, the $\mathrm{NV}^{0}$ centers in the sample experience an atomic environment, which is richer of NV centers and less rich of nitrogen atoms. While from previous reports, the $\mathrm{NV}^{0}$ centers have proved to be resonantly coupled with nitrogen atoms ${ }^{47}$ the data seem to indicate that the resonant coupling from a $\mathrm{NV}^{0}$ to a nearby quenching $\mathrm{NV}^{-}$center is not be very effective. Thus, at least in a small fluence regime, it is reasonable to assume that in low-nitrogen samples, the $\mathrm{NV}^{0}$ centers experience a smaller quenching with increasing fluences. At higher fluences, we can assume that the formation process of $\mathrm{NV}$ centers starts saturating due to the limiting nitrogen concentration: therefore, additional ioninduced defects (vacancy-interstitial pairs and more complex defects) increasingly contribute to the quenching the $\mathrm{NV}^{0}$ emission, thus giving direct evidence that the dipole-dipole energy transfer process between $\mathrm{NV}^{0}$ centers and ion-induced defects is indeed effective.

The $\mathrm{NV}^{-}$emission dataset shown in Fig. 8(b) is not particularly extensive because only at low fluences, was it possible to reliably detect the $\mathrm{NV}^{-}$emission intensity, while at higher fluences, the phononic tail of the $\mathrm{NV}^{0}$ emission tends to overlap with the $\mathrm{NV}^{-}$emission in the spectral detection range [see Fig. 4(a)]. In this rather restricted fluence range, no significant variation of $k$ is measured. This can be explained as due to a weaker coupling of $\mathrm{NV}^{-}$centers to substitutional nitrogen atoms with respect to $\mathrm{NV}^{0}$ centers [see the typical $k$ values in Figs. 8(a) and 8(b), for comparison], as well as to weaker coupling with ion-induced structural defects (at least, in the restricted fluences range reported here).

Similar to what was reported in Fig. 8, Fig. 9 shows the evolution of $k$ for the $\mathrm{NV}^{-}$emission from sample 2 as a function of $2-\mathrm{MeV} \mathrm{H}^{+}$implantation fluence. Also, in this case, mean vacancy density and quantum efficiency values are also reported on additional axes. It is worth stressing that given the higher nitrogen concentration, in sample 2 no reliable signal could be acquired from the $\mathrm{NV}^{0}$ emission, and therefore (different from what was observed in sample 1) the $\mathrm{NV}^{-}$ emission could be reliably detected at higher fluences. From Fig. 9, it is clear how the $k$ value is basically unaffected from implantation conditions, as long as the fluence values are below $\sim 1 \times 10^{16} \mathrm{~cm}^{-2}$ (i.e., average vacancy densities of $\sim 60 \mathrm{ppm}$ ); this is compatible with the low-fluence regime observed for $\mathrm{NV}^{-}$emission also in sample 1 [see Fig. 8(b)]. When damage levels exceed the above-mentioned threshold, $k$ starts increasing with a logarithmic dependence from the fluence. The same type of variation of $k$ is also observed as a function of average damage density, according to the above-mentioned linear approximation. By fitting both the semilogarithmic increase and the plateau at low damage densities due to the background nitrogen concentration, the following relation is

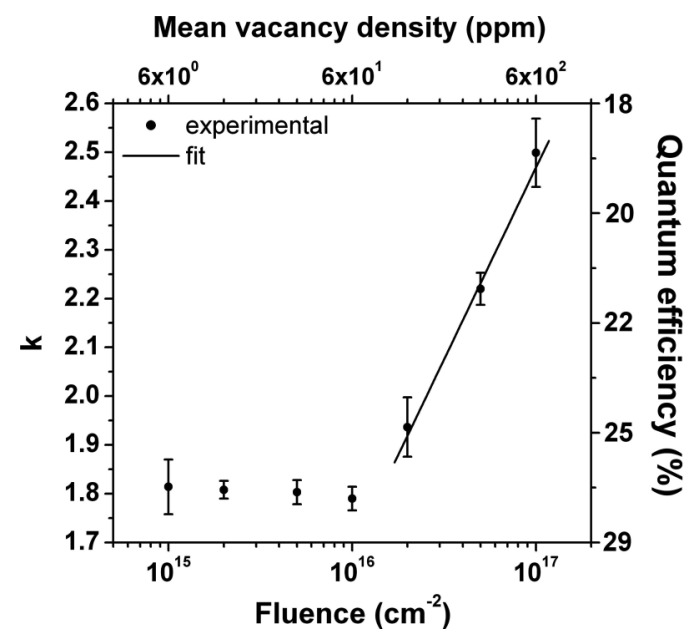

FIG. 9. Evolution of $k$ for the $\mathrm{NV}^{-}$emission from sample 2 as a function of $2-\mathrm{MeV} \mathrm{H}^{+}$implantation fluence. The mean vacancy density across the technique probing depth is reported in the upper horizontal axis, while corresponding quantum efficiency values [as derived from Eq. (3)] are reported on the right-hand vertical axis in a nonlinear scale. Experimental data (dots) are reported together with linear fit of the last four points (line). 
obtained:

$$
k(v)= \begin{cases}(1.803 \pm 0.012) & \text { for } \quad v<60 \mathrm{ppm} \\ (0.411 \pm 0.012)+(0.34 \pm 0.06) \cdot \ln (v[\mathrm{ppm}]) & \text { for } \quad v>60 \mathrm{ppm}\end{cases}
$$

The observed trends are interpreted as follows: at low fluence values the dominating factor in determining a variation in the $\mathrm{NV}^{-}$center lifetime is represented by the concentration of native substitutional nitrogen, while at increasing implantation fluences, the ion-induced damage is the key factor in decreasing the center lifetime. The significant correlation between estimated vacancy densities and $k$ seems to indicate that indeed, at first approximation, isolate vacancy-interstitial defects are the main factor determining $\mathrm{NV}^{-}$centers quenching, although the contribution of more complex defects cannot be ruled out.

\section{Lateral implantations}

As mentioned above, for sample 3, optical probing was performed in an orthogonal geometry with respect to the ion implantations, i.e., parallel direction to the main implanted surface of the sample (see Fig. 2). This approach resulted in an optical measurement, which was directly following the implantation depth, while integrating the signal along a constant vacancy density.

Figure 10 shows a typical TCSPC map acquired in the $(730 \pm 8 \mathrm{~nm})$ spectral window (corresponding to the phonon sideband of the $\mathrm{NV}^{-}$emission) from a region of sample 3 implanted at a fluence of $1 \times 10^{16} \mathrm{~cm}^{-2}$. As reported for sample 2, due to the high nitrogen concentration, no significant

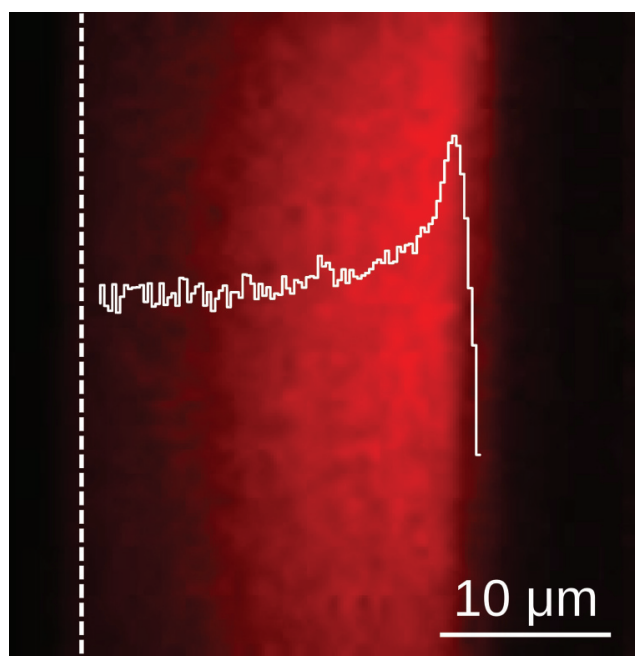

FIG. 10. (Color online) Typical TCSPC map acquired in lateral geometry (see Fig. 2) within the $(730 \pm 8 \mathrm{~nm})$ spectral window (corresponding to the phonon sideband of the $\mathrm{NV}^{-}$emission) from sample 3. The position of the sample surface is highlighted by the vertical dashed line, and increasing depths in the sample bulk span in the horizontal direction towards the right side. The continuous line profile highlights the linear vacancy density profile in semilogarithmic scale reported in Fig. 3 for comparison of SRIM simulations and experimental data.
$\mathrm{NV}^{0}$ emission could be detected in sample 3. The position of the sample surface is highlighted in the figure, and the depth direction is towards the right side of the map. The micrograph is superimposed with the semilogarithmic vacancy density profile reported in Fig. 3 for qualitative comparison of SRIM simulations and experimental data. As expected, Fig. 10 clearly shows how the PL yield increases at increasing depths in the sample bulk, up to the maximum intensity corresponding to the ions end-of-range depth $(\sim 25 \mu \mathrm{m})$. This is clearly because higher vacancy densities in the damage profile correspond to higher NV center concentrations and therefore to stronger PL intensities.

As for frontal implantations, lifetime chronograms have been extracted from the lateral map on a pixel-by-pixel basis, and the resulting fitting $k$ parameters have been averaged across constant depths in the sample (i.e., along vertical lines in the map reported in Fig. 10). The resulting depth profiles of the $k$ values are reported in Fig. 11(a). As for Figs. 8 and 9 , also in this plot the corresponding values of the quantum efficiency [as evaluated with Eq. (3)] are reported on the right vertical axis in a nonlinear scale.

Apart from the direct measurement in lateral geometry, the depth profile of the $k$ value can also be derived from the vacancy density profile obtained from SRIM simulations by applying a variation of Eq. (4) in which the plateau value of $k$ for low damage densities is lower due to the slightly lower nitrogen concentration in this sample as compared to sample 2 (i.e., $190 \mathrm{ppm}$ instead of $200 \mathrm{ppm}$ ). Such a plateau value $(k=1.6)$ was derived for sample 3 from TCSPC measurements in frontal geometry on areas irradiated at the lowest fluences (see the following section for further details). As shown in Fig. 11(a), the directly measured $k$ depth profiles are in good agreement with the above-mentioned numerical predictions (with the possible exception of the data at the very end of range of implanted ions, where the agreement is less satisfactory), thus confirming that the linear approximation in the estimation of vacancy density and the relation reported in Eq. (4) provide an adequate description of the PL quenching process, at least in the damage density ranges reported here.

The measured $k$ values can be correlated with the SRIMderived estimations of the average vacancy density established at different depths in the implanted samples. The resulting plot is reported in a semilogarithmic scale in Fig. 11(b): the observed quasilinear dependence is in good agreement with what was reported in Fig. 9 for frontal implantations. Here, the mismatch between experimental and SRIM data at the ions end of range is reflected in the deviation from linearity at high damage densities.

\section{Low-fluence implantations-effect of nitrogen concentration}

The results of frontal TCSPC measurements of the $\mathrm{NV}^{-}$ emission from the regions implanted at the lowest fluences 
(a)

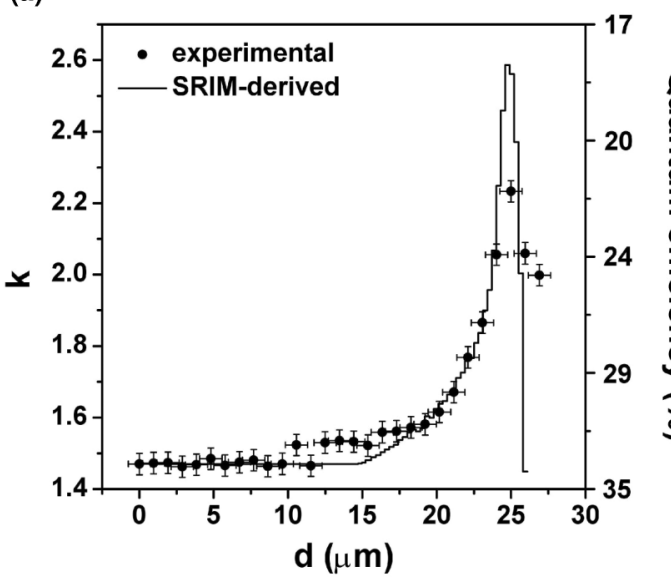

(b)

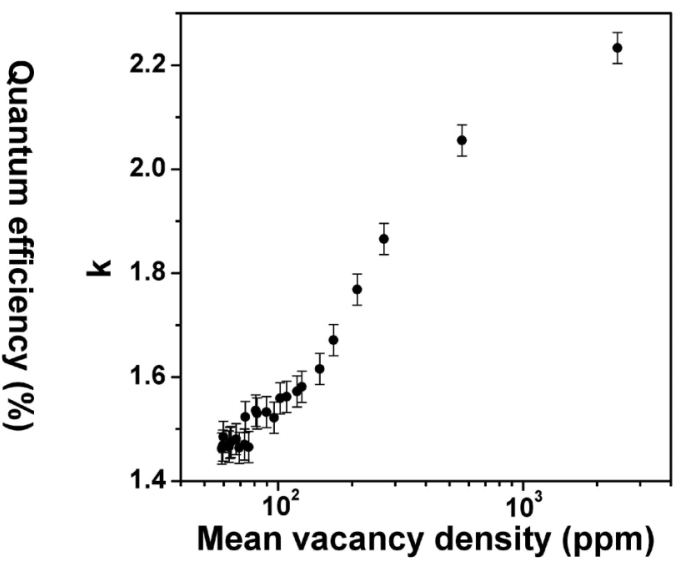

FIG. 11. The $k$ values from a region that was laterally implanted at a fluence of $1 \times 10^{16} \mathrm{~cm}^{-2}$ in sample 3 . (a) $k$ as a function of depth from the sample surface: (dots) experimental data resulting from the chronograms encoded in the TCSPC map reported in Fig. 10 after averaging at constant depths within the sample (i.e., along the vertical axis in Fig. 10); (line) numerical prediction based on SRIM output and Eq. (4); quantum efficiency values [as derived from Eq. (3)] are displayed on the right vertical axis in a nonlinear scale. (b) $k$ as a function of the mean vacancy density at different depths as evaluated with SRIM simulations.

(i.e., $F<3 \times 10^{15} \mathrm{~cm}^{-2}$ ) in all three samples were mutually compared, with the scope of elucidating the effect of native nitrogen on nonradiative coupling in a regime in which the effect of ion-induced damage is negligible (see Figs. 8 and 9). A similar study was not performed for the $\mathrm{NV}^{0}$ emission since a significant native $\mathrm{NV}^{0}$ emission was only measurable in sample 1. Figure 12 shows the variation of the $k$ parameter derived from the fitting of the chronograms acquired in the $(730 \pm 8 \mathrm{~nm})$ spectral range as a function of the substitutional nitrogen concentration estimated with the FTIR measurements (see Sec. II A). The corresponding quantum efficiency, as derived from $k$ through Eq. (3), is also reported on the right vertical axis. The data shown in Fig. 12 are compatible within the reported uncertainties with a linear correlation between $k$

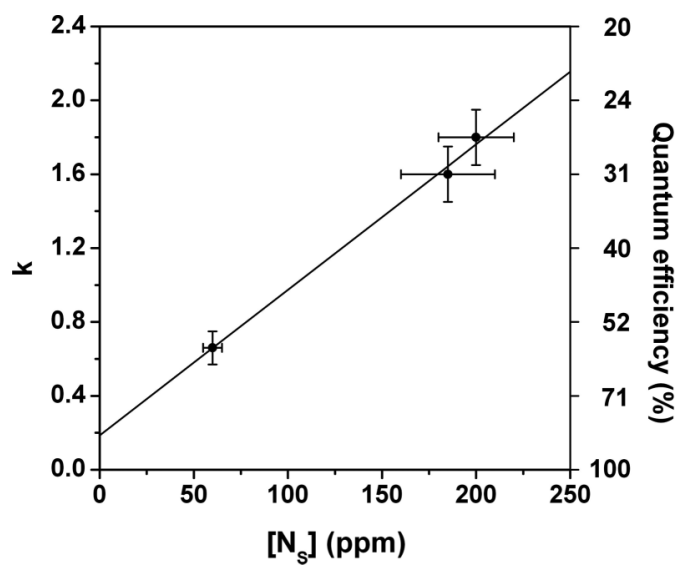

FIG. 12. Variation of the $k$ parameter estimated from the fitting of the chronograms acquired in the $(730 \pm 8 \mathrm{~nm})$ spectral range (corresponding to the phonon sideband of the $\mathrm{NV}^{-}$emission) from samples 1-3 prior to ion implantation, as a function of substitutional nitrogen concentration estimated with FTIR measurements. On the right vertical axis, the corresponding quantum efficiency values [see Eq. (3)] are reported. Experimental data with uncertainties (dots) are reported with the linear fitting curve (line). and $\left[N_{S}\right]$. The resulting fitting curve for $k$ vs $\left[N_{S}\right]$ is

$$
k=(2 \pm 1) \times 10^{-1}+(8 \pm 1) \times 10^{-3} \cdot\left[N_{S}\right] .
$$

It is worth noting that in the result above, a significant contribution to the uncertainty of the obtained parameters is propagated from the uncertainty on the value of the radiative lifetime. Having said that, it can be observed that, since the intercept value in the linear regression is affected by a significant uncertainty, it is not possible to rule out that a nonzero $k$ value is observed in an ideal sample with no substitutional nitrogen and therefore that other kinds of native defects affect the $\mathrm{NV}^{-}$lifetime. It is worth mentioning that for $\left[N_{S}\right] \cong 100 \mathrm{ppm}$ (a value which is typical for type Ib samples produced with the HPHT technique), the linear fit yields a $k$ value corresponding to a quantum efficiency of $\eta \cong 50 \%$ through the application of Eq. (3). Undoubtedly, this kind of analysis would benefit from the characterization of a larger set of samples characterized by a broader spectrum of $\left[N_{S}\right]$, but this goes beyond the scope of the present work.

\section{CONCLUSIONS}

A TCSPC microscopy technique was successfully used to characterize the lifetime of $\mathrm{NV}^{-}$and $\mathrm{NV}^{0}$ centers in type $\mathrm{Ib}$ HPHT single-crystal samples with different concentrations of native substitutional nitrogen, after systematic $2-\mathrm{MeV} \mathrm{H}^{+}$ion implantation in different geometries and at increasing fluences. The obtained results can be summarized as follows:

(1) while a radiative lifetime for $\mathrm{NV}^{-}$centers of (12 $\left.\pm 1 \mathrm{~ns}\right)$ was taken from the literature, ${ }^{46}$ the radiative lifetime for $\mathrm{NV}^{0}$ centers was directly measured in an optical-grade $\mathrm{N}$-implanted sample, yielding a value of $(17 \pm 1 \mathrm{~ns})$, in satisfactory agreement with the literature; ${ }^{47}$

(2) the dipole-dipole resonant energy transfer was successfully applied to model the measured decay times of $\mathrm{NV}^{0}$ and $\mathrm{NV}^{-}$centers in different experimental conditions, allowing the determination of empirical parameter $k$ and accounting for the 
strength of the nonradiative coupling between the PL centers under investigation and native/induced defects in the samples;

(3) in frontally implanted samples, the variation of the $k$ parameter was reported as a function of ion-induced defect density for both $\mathrm{NV}^{0}$ and $\mathrm{NV}^{-}$emissions: while a nonmonotonic variation was observed for $\mathrm{NV}^{0}$ emission, a logarithmic increase of $k$ vs damage density was found for $\mathrm{NV}^{-}$emission for damage levels exceeding a critical threshold determined by the background nitrogen concentration; the data relevant to $\mathrm{NV}^{-}$emission were quantitatively analyzed;

(4) in laterally implanted samples, it was possible to map the evolution of the PL lifetime across the sample thickness, thus obtaining direct evidence of the effects of the strongly nonuniform damage profile; the experimental data exhibited a good agreement with numerical prediction based on SRIM simulations combined with the results of the quantitative analysis carried out for frontally implanted samples;

(5) in regions implanted at the lowest fluences, a linear dependence was identified between native nitrogen substitutional concentration independently measured by FTIR spectroscopy and the $k$ value; this allowed the attribution of the quenching of $\mathrm{NV}^{-}$emission primarily to nitrogen, although other kinds of native defects cannot be ruled out in principle.

Finally, it is worth noting that the evaluation of the empirical parameter $k$ allows the estimation of the mean quantum efficiency of the luminescence centers, which is a physical property of extreme relevance for the current studies on the use of diamond color centers for photonic applications, particularly with regard to those fabrication-functionalization processes involving ion beam irradiation.

\section{ACKNOWLEDGMENTS}

We thank J. Meijer for the $10-\mathrm{MeV} \mathrm{N}$ implantations performed at the "heavy ion leg" beam of the $4 \mathrm{MV}$ Tandem accelerator of the RUBION laboratories (Ruhr Universität Bochum). This work is supported by the following projects and grants, which are gratefully acknowledged: FIRB "Future in Research 2010" Project (CUP code: D11J11000450001) funded by the Italian Ministry for Teaching, University and Research (MIUR); "Dia.Fab.” experiment at the INFN Legnaro National Laboratories; one-month DAAD 2011 type-1B grant "Ion implantation in diamond for applications in photonics" (application number: A/11/78148) funded by the German Academic Exchange Service; "A.Di.N-Tech." project (CUP code: D15E13000130003) funded by University of Torino and Compagnia di San Paolo in the framework of the "Progetti di ricerca di Ateneo 2012" scheme; University of TorinoCompagnia di San Paolo Project No. ORTO11RRT5, 2011, Linea $1 \mathrm{~A}$.
*Present address: Nokia Research Center, Cambridge, United Kingdom.

${ }^{\dagger}$ Present address: European XFEL GmbH, Hamburg, Germany.

${ }^{\ddagger}$ Corresponding author: paolo.olivero@unito.it

${ }^{1}$ W. Yang, Z. Xu, M. Feng, and J. Du, New J. Phys. 12, 113039 (2010).

${ }^{2}$ G. Waldherr, P. Neumann, S. F. Huelga, F. Jelezko, and J. Wrachtrup, Phys. Rev. Lett. 107, 090401 (2011).

${ }^{3}$ L. Dai and L. C. Kwek, Phys. Rev. Lett. 108, 066803 (2012).

${ }^{4}$ E. Togan, Y. Chu, A. Imamoglu, and M. D. Lukin, Nature (London) 478, 497 (2011).

${ }^{5}$ G. D. Fuchs, G. Burkard, P. V. Klimov, and D. D. Awschalom, Nat. Phys. 7, 789 (2011).

${ }^{6}$ X. Zhu, S. Saito, A. Kemp, K. Kakuyanagi, S. Karimoto, H. Nakano, W. J. Munro, Y. Tokura, M. S. Everitt, K. Nemoto, M. Kasu, N. Mizuochi, and K. Semba, Nature (London) 478, 221 (2012).

${ }^{7}$ P. Maletinsky, S. Hong, M. S. Grinolds, B. Hausmann, M. D. Lukin, R. L. Walsworth, M. Loncar, and A. Yacoby, Nat. Nanotechnol. 7, 320 (2012).

${ }^{8}$ N. M. Nusran, M. Ummal Momeen, and M. V. Gurudev Dutt, Nat. Nanotechnol. 7, 109 (2012).

${ }^{9}$ G. Waldherr, J. Beck, P. Neumann, R. S. Said, M. Nitsche, M. L. Markham, D. J. Twitchen, J. Twamley, F. Jelezko, and J. Wrachtrup, Nat. Nanotechnol. 7, 105 (2012).

${ }^{10}$ T. Choy, B. J. M. Hausmann, T. M. Babinec, I. Bulu, M. Khan, P. Maletinsky, A. Yacoby, and M. Loncar, Nat. Photonics 5, 738 (2011).

${ }^{11}$ A. Lohrmann, S. Pezzagna, I. Dobrinets, P. Spinicelli, V. Jacques, J.-F. Roch, J. Meijer, and A. M. Zaitsev, Appl. Phys. Lett. 99, 251106 (2011).
${ }^{12}$ N. Mizuochi, T. Makino, H. Kato, D. Takeuchi, M. Ogura, H. Okushi, M. Nothaft, P. Neumann, A. Gali, F. Jelezko, J. Wrachtrup, and S. Yamasaki, Nat. Photonics 6, 299 (2012).

${ }^{13}$ O. Arcizet, V. Jacques, A. Siria, P. Poncharal, P. Vincent, and S. Seidelin, Nat. Phys. 7, 879 (2011).

${ }^{14}$ S. Hong, M. S. Grinolds, P. Maletinsky, R. L. Walsworth, M. D. Lukin, and A. Yacoby, Nano Lett. 12, 3920 (2012).

${ }^{15}$ S. Kolkowitz, A. C. Bleszynski Jayich, Q. P. Unterreithmeier, S. D. Bennett, P. Rabl, J. G. E. Harris, and M. D. Lukin, Science 335, 1603 (2012).

${ }^{16}$ F. C. Waldermann, P. Olivero, J. Nunn, K. Surmacz, Z. Y. Wang, D. Jaksch, R. A. Taylor, I. A. Walmsley, M. Draganski, P. Reichart, A. D. Greentree, D. N. Jamieson, and S. Prawer, Diamond Relat. Mater. 16, 1887 (2007).

${ }^{17}$ B. Naydenov, F. Reinhard, A. Lämmle, V. Richter, R. Kalish, U. F. S. D’Haenens-Johansson, M. Newton, F. Jelezko, and J. Wrachtrup, Appl. Phys. Lett. 97, 242511 (2010).

${ }^{18}$ A. Dréau, J.-R. Maze, M. Lesik, J.-F. Roch, and V. Jacques, Phys. Rev. B 85, 134107 (2012).

${ }^{19}$ T. Ishikawa, K.-M. C. Fu, C. Santori, V. M. Acosta, R. G. Beausoleil, H. Watanabe, S. Shikata, and K. M. Itoh, Nano Lett. 12, 2083 (2012).

${ }^{20}$ N. Zhao, J.-L. Hu, Sai-Wah Ho, J. T. K. Wan, and R. B. Liu, Nat. Nanotechnol. 6, 242 (2011).

${ }^{21}$ X. F. He, N. B. Manson, and P. T. H. Fisk, Phys. Rev. B 47, 8816 (1993).

${ }^{22}$ R. J. Epstein, F. M. Mendoza, Y. K. Kato, and D. D. Awschalom, Nat. Phys. 1, 1 (2005).

${ }^{23}$ I. Aharonovich, C. Santori, B. A. Fairchild, J. Orwa, K. Ganesan, K.-M. C. Fu, R. G. Beausoleil, A. D. Greentree, and S. Prawer, J. Appl. Phys. 106, 124904 (2009). 
${ }^{24}$ B. Naydenov, V. Richter, J. Beck, M. Steiner, P. Neumann, G. Balasubramanian, J. Achard, F. Jelezko, J. Wrachtrup, and R. Kalish, Appl. Phys. Lett. 96, 163108 (2010).

${ }^{25}$ I. Bayn, A. Bolker, C. Cytermann, B. Meyler, V. Richter, J. Salzman, and R. Kalish, Appl. Phys. Lett. 99, 183109 (2011).

${ }^{26}$ J. Botsoa, T. Sauvage, M.-P. Adam, P. Desgardin, E. Leoni, B. Courtois, F. Treussart, and M.-F. Barthe, Phys. Rev. B 84, 125209 (2011).

${ }^{27}$ Y. Deshko, M. Huang, and A. A. Gorokhovsky, J. Lumin. 131, 489 (2011).

${ }^{28}$ J. Schwartz, P. Michaelides, C. D. Weis, and T. Schenkel, New J. Phys. 13, 035022 (2011)

${ }^{29}$ J. O. Orwa, C. Santori, K. M. C. Fu, B. Gibson, D. Simpson, I. Aharonovich, A. Stacey, A. Cimmino, P. Balog, M. Markham, D. Twitchen, A. D. Greentree, R. G. Beausoleil, and S. Prawer, J. Appl. Phys. 109, 083530 (2011).

${ }^{30}$ R. Kalish, Nucl. Instrum. Methods Phys. Res. B 272, 42 (2012).

${ }^{31}$ L. Childress, M. V. Gurudev Dutt, J. M. Taylor, A. S. Zibrov, F. Jelezko, J. Wrachtrup, P. R. Hemmer, and M. D. Lukin, Science 314, 281 (2006).

${ }^{32}$ R. Hanson, V. V. Dobrovitski, A. E. Feiguin, O. Gywat, and D. D. Awschalom, Science 320, 352 (2008).

${ }^{33}$ J. Du, X. Rong, N. Zhao, Y. Wang, J. Yang, and R. B. Liu, Nature (London) 461, 1265 (2009).

${ }^{34}$ G. de Lange, Z. H. Wang, D. Ristè, V. V. Dobrovitski, and R. Hanson, Science 330, 60 (2010).

${ }^{35}$ C. A. Ryan, J. S. Hodges, and D. G. Cory, Phys. Rev. Lett. 105, 200402 (2010).

${ }^{36}$ B. Naydenov, F. Dolde, L. T. Hall, C. Shin, H. Fedder, L. C. L. Hollenberg, F. Jelezko, and J. Wrachtrup, Phys. Rev. B 83, 081201 (2011).

${ }^{37}$ Z.-H. Wang, G. de Lange, D. Ristè, R. Hanson, and V. V. Dobrovitski, Phys. Rev. B 85, 155204 (2012).

${ }^{38}$ M. Inokuti and F. Hirayama, J. Chem. Phys. 43, 1978 (1965).

${ }^{39} \mathrm{~W}$. R. Ware, in Creation and detection of the excited states, edited by A. A. Lamola, Vol. 1, Part A (Marcel Dekker Inc, New York, 1971), pp. 213-302.

${ }^{40}$ G. Liaugaudas, A. T. Collins, K. Suhling, G. Davies, and R. Heintzmann, J. Phys.: Condens. Matter 21, 364210 (2009).

${ }^{41}$ M. D. Crossfield, G. Davies, A. T. Collins, and E. C. Lightowlers, J. Phys. C 7, 1909 (1974).
${ }^{42}$ M. F. Thomaz and G. Davies, Proc. R. Soc. London, Sect. A 362, 405 (1978).

${ }^{43}$ A. T. Collins, M. F. Thomaz, and M. I. B. Jorge, J. Phys. C 16, 2177 (1983).

${ }^{44} \mathrm{G}$. Davies, Properties and Growth of Diamond (INSPEC, London, 1994).

${ }^{45}$ L. Robledo, H. Bernien, T. Van der Sar, and R. Hanson, New J. Phys. 13, 025013 (2011).

${ }^{46}$ A. Batalov, C. Zierl, T. Gaebel, P. Neumann, I.-Y. Chan, G. Balasubramanian, P. R. Hemmer, F. Jelezko, and J. Wrachtrup, Phys. Rev. Lett. 100, 077401 (2008).

${ }^{47}$ G. Liaugaudas, G. Davies, K. Suhling, R. U. A. Khan, and D. J. F. Evans, J. Phys.: Condens. Matter 24, 435503 (2012).

${ }^{48}$ W. J. P. van Enckevort and H. G. M. Lochs, J. Appl. Phys. 64, 434 (1988).

${ }^{49}$ A. Yelisseyev, V. Nadolinny, B. Feigelson, S. Terentyev, and S. Nosukhin, Diam. Relat. Mater. 5, 1113 (1996).

${ }^{50}$ V. S. Drumm, A. C. Alves, J. O. Orwa, D. N. Jamieson, J. C. McCallum, S. Prawer, and C. C. G. Ryan, Phys. Status Solidi A 208, 42 (2010).

${ }^{51}$ R. M. Chrenko, H. M. Strong, and R. E. Tuft, Philos. Mag. 182, 313 (1971).

${ }^{52}$ J. F. Ziegler, J. P. Biersack, and M. D. Ziegler, SRIM-The Stopping and Range of Ions in Matter (Ion Implantation Press, Mormsville, NC, 2008).

${ }^{53}$ W. Wu and S. Fahy, Phys. Rev. B 49, 3030 (1994).

${ }^{54}$ A. M. Zaitsev, Optical Properties of Diamond: A Data Handbook (Springer, New York, 2001).

${ }^{55}$ A. T. Collins, J. Phys.: Condens. Matter 14, 3743 (2002).

${ }^{56} \mathrm{~W}$. Becker, The bh TCSPC Handbook, 4th ed. (Becker \& Hickl $\mathrm{GmbH}$, Berlin, 2010).

${ }^{57}$ T. Förster, Ann. Phys. 2, 55 (1948).

${ }^{58}$ Y. Mita, Phys. Rev. B 53, 11360 (1996).

${ }^{59}$ J. W. Steeds, T. J. Davis, S. J. Charles, J. M. Hayes, and J. E. Butler, Diam. Relat. Mater. 8, 1847 (1999).

${ }^{60}$ J. Harrison, M. J. Sellars, and N. B. Manson, J. Lumin. 107, 245 (2004).

${ }^{61}$ J. Harrison, M. J. Sellars, and N. B. Manson, Diamond Relat. Mater. 15, 586 (2006).

${ }^{62}$ G. D. Fuchs, V. V. Dobrovitski, D. M. Toyli, F. J. Heremans, C. D. Weis, T. Schenkel, and D. D. Awschalom, Nat. Phys. 6, 668 (2010). 